\title{
Cómo perciben los hombres tener diabetes mellitus tipo 2: casos en Bogotá*
}

How men perceive having type 2 diabetes mellitus: cases in Bogota

Como os homens percebem ter diabetes mellitus tipo 2: casos em Bogotá

Lorena Chaparro-Diaz ${ }^{\mathrm{a}}$

Universidad Nacional de Colombia, Colombia

DOI: https://doi.org/10.11144/Javeriana.ie22.cpht

olchaparrod@unal.edu.co

ORCID: https://orcid.org/0000-0001-8241-8694

Recibido: 08 Abril 2019

Geu Mendoza-Catalán

Aceptado: 26 Marzo 2020

Universidad Autónoma de Baja California, México

Publicado: 22 Julio 2020

ORCID: https://orcid.org/0000-0002-5061-2457

Sonia Carreño Moreno

Universidad Nacional de Colombia, Colombia

ORCID: https://orcid.org/0000-0002-4386-6053

Juana Mercedes Gutiérrez-Valverde

Universidad Autónoma de Nuevo León, México

ORCID: https://orcid.org/0000-0001-9506-5947

\section{Resumen:}

Introducción: La diabetes mellitus es una enfermedad crónica que se percibe y se maneja de distintas maneras según las concepciones que cada persona tiene y que pueden ser diferentes según el género. Objetivo: Describir la percepción de estar enfermo de los hombres con diabetes mellitus tipo 2 en población colombiana. Método: Estudio cualitativo-descriptivo con análisis de contenido basado en una categorización de tipo inductivo. Se incluyeron 6 participantes que cumplieron con los criterios de inclusión de ser hombres mayores de 30 años con diagnóstico de diabetes mellitus tipo 2 y con una duración de la enfermedad de al menos un año, durante 2016. Se realizaron entrevistas semiestructuradas sobre su proceso y percepción de la enfermedad en su vida, las cuales se analizaron y codificaron por medio del programa Atlas.ti. Resultados: Las 7 categorías obtenidas dan cuenta de la percepción de los hombres respecto a la enfermedad en la que asocian la salud con ausencia de síntomas y dolor, poder entender lo que era tener diabetes implicó un proceso de afrontamiento y adaptación en el que buscaron darle sentido a la diabetes a través conocer qué es la enfermedad y cómo incorporarla a la vida cotidiana Conclusión: La vivencia de la diabetes en los hombres adultos mayores colombianos representa las dificultades para aceptar el diagnóstico, comprometerse con comportamientos de autocuidado adecuados para el control de la diabetes y reconocer y buscar ayuda asistencial cuando la requieren por complicaciones de la enfermedad.

Palabras clave: apoyo social, cuidadores, enfermedad crónica, carga de la enfermedad, cuidado de enfermería.

\section{Abstract:}

Introduction: Diabetes mellitus is a chronic disease that is perceived and managed in different ways according to the notions that each person has, which may be different based on gender. Objective: To describe the perception of men on having type 2 diabetes mellitus across the Colombian population. Method: Qualitative-descriptive study with content analysis based on an inductive categorization. Six participants who met the inclusion criteria (men, older than 30 years of age, with a diagnosis of type 2 diabetes mellitus, and with a duration of the disease of at least one year in 2016) were included. Semi-structured interviews were conducted on their process and perception of the disease in their lives, which were then analyzed and coded using the Atlas.ti program. Results: The 7 categories obtained account for the perception of men regarding the disease. These perceptions show an association of health with the absence of symptoms and pain. To understand what having diabetes was like implied a process of coping and adaptation in which they sought to make sense out of diabetes through knowing what the disease is and how to integrate it into daily life Conclusion: The experience of diabetes in older Colombian men shows the difficulty inherent to accepting the diagnosis,

Notas de autor

\footnotetext{
${ }^{\text {a }}$ Autora de correspondencia. Correo electrónico: olchaparrod@unal.edu.co
} 
committing to self-care behaviors adequate for the control of diabetes, and recognizing and seeking health care when required for complications of the disease.

Keywords: social support, caregivers, chronic illness, burden of disease, nursing care.

\section{Resumo:}

Introdução: O diabetes mellitus é uma doença crônica percebida e tratada de diferentes maneiras de acordo com as concepções de cada pessoa e que podem ser diferentes segundo o gênero. Objetivo: Descrever a percepção de estar doente em homens com diabetes mellitus tipo 2 na população colombiana. Método: Estudo qualitativo-descritivo com análise de conteúdo baseado em uma categorização de tipo indutivo. Incluíram-se seis participantes que atenderam os critérios de inclusão de serem homens com mais de 30 anos, com diagnóstico de diabetes mellitus tipo 2 e com duração da doença de ao menos um ano, durante 2016. Foram realizadas entrevistas semiestruturadas sobre o processo e percepção da doença na vida deles, as quais foram analisadas e codificadas com o programa Atlas.ti. Resultados: As sete categorias obtidas explicam a percepção dos homens em relação à doença na que associam saúde com ausência de sintomas e dor, conseguir entender o que era ter diabetes envolveu um processo de enfrontamento e adaptação no que visaram lhe dar senso à diabetes através de conhecer o que a doença é e como incorporá-la à vida cotidiana Conclusão: A vivência do diabetes em homens adultos maiores colombianos representa as dificuldades para aceitar o diagnóstico, se comprometer com comportamentos de autocuidado adequados para o controle do diabetes e reconhecer e procurar ajuda assistencial quando necessário por complicações da doença.

Palavras-chave: suporte social, cuidadores, doença crônica, ônus da doença, cuidado de enfermagem.

\section{Introducción}

La diabetes mellitus (DM) es una enfermedad crónica responsable de altas tasas de mortalidad y carga de discapacidad en el mundo, tanto que para el 2016 se estimó que ocurrieron 1,5 millones de muertes causadas directamente por la DM, el 80 \% en países de medianos y bajos ingresos $(1,2)$. En Colombia, la prevalencia de la DM es del 7,03 \% en la población, y solo el 50 \% recibe tratamiento médico. De ahí que se considere una las primeras 10 causas de mortalidad general (3-5).

Dentro de las acciones para prevenir y reducir los rezagos de la DM, se encuentran la búsqueda de atención en salud, los cambios de estilo de vida y la adherencia al tratamiento. Algunos estudios (6-10) han indicado que dentro del comportamiento de las enfermedades crónicas existen algunas diferencias marcadas por el género frente al cuidado, la adherencia y la búsqueda de ayuda. Así, los hombres son quienes frecuentemente evitan la asistencia médica, ocultan su diagnóstico y prestan poca atención a las recomendaciones; por lo que la aparición de complicaciones es más alta en esta población.

A pesar de que se han señalado diferentes factores personales, psicosociales, culturales y religiosos que ayudan y limitan la búsqueda de atención médica y la adherencia al tratamiento, la forma como las personas se perciben sanos o enfermos pudiera ser un indicador que ayude a la toma de decisiones sobre su futuro próximo. Al hacer una diferenciación con el género, la concepción de salud puede variar, incluso en la actualidad cuando existen diferentes definiciones formales del concepto salud. Así es como algunos autores concuerdan respecto a que en los hombres la concepción de salud tiende a ser más específica en cuanto a presencia de síntomas o sensaciones que le generan malestar, siendo esta una definición más práctica al ir relacionado con sus creencias (7,10-14).

Por lo planteado, es importante profundizar en la forma como los hombres con DM interpretan estar enfermos para buscar atención y realizar actividades de autocuidado, teniendo en cuenta que en un inicio la enfermedad puede ser asintomática y que la presencia de síntomas severos se da cuando se han desarrollado complicaciones prevenibles. Así, los resultados de esta investigación permiten apreciar cómo es la percepción de sentirse enfermo cuando ya se tiene un diagnóstico de DM y cómo esta percepción puede influir en la decisión de cuidarse y seguir las recomendaciones médicas.

Para enfermería comprender esa comunicación, interrelación y comprensión del mundo en que las personas se desarrollan, sus significados y el sentido que estos les dan, los cuales influyen en la adopción de conductas en 
la vida diaria (15), permitirá orientar a los usuarios a comprender mejor su enfermedad, ayudar en la toma de decisiones y favorecer la adherencia. En consecuencia, el objetivo de este estudio fue describir la percepción de estar enfermo en hombres con DM.

\section{Materiales y métodos}

Este fue un estudio de abordaje cualitativo-descriptivo que utiliza como metodología el análisis de contenido de tipo inductivo y permite identificar actitudes, creencias, valores, percepciones, metas y otros aspectos de un grupo de personas $(16,17)$, en este caso los hombres con DM tipo 2 frente a su enfermedad. Los sujetos de investigación fueron 6 participantes que cumplieron con los criterios de inclusión de ser hombres con DM tipo 2, mayores de 30 años de edad y con una duración de la enfermedad de al menos un año. Ellos fueron captados en centros de atención primaria y secundaria en Bogotá (Colombia) durante el 2016. Se utilizó el muestreo teórico hasta obtener saturación de los datos, que tuvo los criterios de contar con las dimensiones, las propiedades y las subpropiedades derivadas de los datos. Otro criterio fue realizar segundas entrevistas con preguntas adicionales a las iniciales de acuerdo con las necesidades de información detectadas en los primeros resultados, identificando los vacíos que aún estaban presentes en el análisis de los datos y así lograr saturar cada categoría $(18,19)$.

Frente al proceso de recolección de los datos, uno de los investigadores abordó a las personas que se encontraban en la sala de espera antes a pasar a su consulta, previa indicación del personal de salud sobre aquellos hombres que cumplían con los criterios de inclusión, se les explicó el objetivo de la investigación y, posteriormente, se les señaló el interés de realizar una entrevista. Si la persona aceptaba, se realizaba la firma del consentimiento informado y se determinaba el momento y lugar. La institución de salud donde se captaron los participantes otorgó un lugar que garantizó la privacidad y se desarrolló la entrevista semiestructurada (20) con las siguientes opciones iniciales de preguntas: como hombre, ¿ puede explicarme cómo se da cuenta de que se encuentra enfermo? ¿Qué significado tiene estar enfermo para usted? ¿Cómo cuida usted de su salud? ¿Estar enfermo afecta su identidad como hombre? ¿Cuáles son los pensamientos que tiene usted cuando se siente enfermo? Derivado de las respuestas a estas preguntas, el investigador iba estableciendo preguntas específicas para cada participante, especialmente cuando se requería ampliar información o indagar a profundidad las razones de las afirmaciones que podía hacer.

Esta guía de preguntas no constituye un instrumento de recolección de datos, tal como se concibe en el paradigma cuantitativo; esta es una entrevista cualitativa desarrollada por uno de los investigadores y verificada por el otro investigador, quien cuenta con amplia experiencia en estudios de abordaje cualitativo y cuidado de personas con enfermedades crónicas no transmisibles.

Estas seis entrevistas cualitativas se grabaron en audio y se transcribieron, para analizarlas posteriormente mediante las pautas que indica el análisis de contenido, como lo plantea Krippendorff (21), de quien se tomaron los siguientes pasos:

1. Formulación de los datos: determinación de unidades de análisis.

2. Reducción de datos.

3. Inferencia de códigos y categorías.

4. Análisis y validación de los datos.

Para el análisis se recopilaron los documentos transcritos en el software Atlas.ti versión 7.5.4., donde se creó una Unidad Hermenéutica con cada entrevista transcrita en la que se identificaron los códigos descriptores. Después de etiquetado cada código descriptor, se agruparon los códigos en categorías y subcategorías. Cada uno de los investigadores analizó críticamente el proceso de codificación y categorización, dándole relevancia a la reflexividad sobre las concepciones de cada uno respecto a este fenómeno y así dejarlas de lado durante 
el análisis. Finalmente, cada categoría se interpretó y confirmó tanto por los participantes como por los investigadores, es decir, se dio cumplimiento a los criterios de rigor de credibilidad y confirmabilidad (Guba 22), en el que lo que cada investigador analizó se triangula con el otro (se cruzan datos) y se presenta a los participantes para que confirmen si se identifican o no con las categorías presentadas. Para esta investigación se obtuvo la validación de tres hombres participantes.

Esta investigación cumplió con los criterios éticos de respeto, beneficencia y confidencialidad. Contó con aval del Comité de Ética en la Facultad de Enfermería de la Universidad Nacional de Colombia y autorización de centros de atención primaria u hospitalaria. Todos los participantes firmaron el consentimiento informado de manera voluntaria.

\section{Resultados}

Este estudio contó con la participación de 6 hombres, con una edad promedio de 69,6 años (DE = 11,7), en un rango de 49 a 81 años, quienes llevaban entre 4 y 25 años con DM. La escolaridad estuvo entre primaria y profesional. Del estado civil, 5 eran casados y uno viudo. Del total de los hombres, uno se encontraba empleado, el resto eran pensionados o sin vinculación laboral. La mayoría mencionó profesar religión católica (4) o cristiana (2). El grado de compromiso religioso se reportó en un nivel medio (3) y alto (3). De acuerdo con el lugar de procedencia, correspondían a los departamentos de Bogotá (2), Boyacá (2), Cundinamarca (1) y Bucaramanga (1).

Cuatro hombres tenían DM e hipertensión arterial y 2 tenían solo DM. El tiempo con la enfermedad en promedio fue de 14,6 años $(\mathrm{DE}=7,6)$, en un rango de 4 a 25 años. Todos los participantes tenían baja dependencia y un nivel cognitivo intacto. Cinco participantes señalaron tener un único cuidador y todos los hombres señalaron que su cuidador primario era una mujer, principalmente su esposa, seguido de la hermana o hija, con edad promedio en las cuidadoras de 59,1 años $(\mathrm{DE}=12,0)$ y entre 21 y 68 años. Todos se habían radicado en la ciudad de Bogotá y hacían parte del régimen contributivo del sistema de salud colombiano. A continuación se detallan cada una de las categorías con sus respectivas descripciones y expresiones de los participantes.

\section{Automanejo y autogestión de la enfermedad}

Para los participantes, la DM — como enfermedad crónica - requiere inicialmente aprender sobre ella, es decir, estos hombres, luego del diagnóstico, se tomaron el tiempo de conocer la enfermedad, con el fin de idear estrategias para realizar cambios en su estilo de vida, como expresa uno de sus participantes:

Yo pensaba que uno se recuperaba y que no volvía a molestar, pero entonces como dicen que el páncreas como que se le acaba uno, deja de funcionar, entonces yo me fui dando cuenta que necesitaba tomarme la droga, que necesitaba estar obedeciendo al médico, ser juicioso con mi cuidado. (P01-22)

Así, ellos reconocen y saben que la DM es una enfermedad que requiere un control estricto frente al manejo de las concentraciones de glucemia, lo que se logra por medio de una alimentación saludable y una vida activa. Así, para los participantes se hace indispensable el apoyo familiar para lograr su autocuidado y controlar la enfermedad:

Yo tengo una hermana que es bacterióloga y ella también me explicó que no debía volver a comer chocolate, ni a comer ni dulce ni nada, entonces aquí mi nuera es la que cocina, cocina con poca grasa, y cocina bajito de sal, así todo bien y entonces yo almuerzo y me siento bien. (P01-15)

Por otra parte, los hombres participantes reconocen que han tenido algunas falencias frente a la gestión de su cuidado y de su enfermedad, debido a que muchas veces les es difícil adherirse a todas las recomendaciones terapéuticas que reciben por parte de los profesionales de la salud. Sumado a esto, también refieren que al no 
identificar síntomas que podrían ser desagradables, creen que la enfermedad está bajo control, aun cuando esto no es así:

No me gusta tomar el medicamento, pero sí trato de llevar por la mañana una pasta para después del desayuno, y trato de tomarla, porque nunca me ha gustado tomar pastas y aunque a veces me como mis harinas, como pasta, como plátano, todo de a poquito, a mí no me da lo que dicen, por ejemplo cortarse, dicen que los diabéticos no se deben de cortar porque no les cicatrizan las heridas, pero a mí eso no me da, no me afecta. (P03-66)

En general, los participantes expresan su preocupación e interés en torno a aspectos del autocuidado, pues reconocen que, a pesar del conocimiento que tienen de la enfermedad, les es difícil dejar de lado comportamientos habituales; sin embargo, el apoyo familiar les ha facilitado la gestión de su propio cuidado.

\section{Concepción de salud y bienestar}

Para los hombres participantes, la DM no representa un obstáculo para el desarrollo de su vida cotidiana. Así, para ellos el estar sanos y tener bienestar dentro de su proceso de enfermedad implica el aprender a controlarla. Adicionalmente, los participantes refieren que poder seguir con su vida laboral los hace sentir productivos, y para ellos esto representa el estar saludable: "A uno le toca seguir lo que el médico le dice: que de la comida, y el azúcar y la bebida (alcohólica) y caminar aunque sea una hora diaria, eso hace preciso que esté uno bien, y así también uno pueda trabajar" (P04-49).

Para otros, el estar sano se relaciona con la ausencia de síntomas, es decir, que si ellos perciben que su cuerpo no presenta cambios que los haga sentir incómodos, con molestia o con dolor, su percepción de bienestar es adecuada. Esta ausencia de síntomas lleva a que ellos expresen que no sienten temor por la enfermedad, debido a sus características, en las que sus complicaciones se ven a largo plazo y no se manifiestan de manera abrupta:

Pues, que no le diera a uno esa vaina, que ponerse uno a sudar y sentirse desalentado, si no sintiera eso me sentiría sano, me sentiría muy bien. (P04-51)

El no sentir ningún dolor es sentirse como alentado, uno que tiene esta enfermedad, así no le da temor porque uno se siente bien (P02-13)

\section{Afrontamiento y adaptación a la enfermedad}

Ante el diagnóstico de DM, los participantes refieren el desarrollo de diferentes estrategias para enfrentar la enfermedad y adaptarse a los cambios. Al principio, estos hombres intentan buscar una explicación a sus síntomas, diferentes al diagnóstico, evidenciando en una forma de negación y evitación de la enfermedad. Durante este proceso algunos síntomas empeoran, de modo que deben enfrentar las consecuencias; con esto, los participantes adquieren conocimientos para aprender a vivir con la diabetes:

Si hay veces que sí me ha pasado eso [síntomas desagradables] y no les hago caso y pienso que es algo pasajero, y a veces que le da a uno un mareo o cualquier cosa, pienso que algo me hizo daño, pero no como para ir al médico y menos por cualquier cosa que le pase a uno, yo pienso que toda enfermedad es como psicológica, entonces es mejor no pensar en la enfermedad que estar pensando en ella. Por eso cuando voy al médico y ve que estoy en 600 ya me quiere dejar hospitalizado, y le digo que no, y digo que no quiero insulina, ni hospitalizarme. (P03-99)

En este proceso no se puede dejar de lado sus creencias, en este caso la creencia en el tratamiento y en un ser superior. El aferrarse a esto demuestra su deseo por vivir y por lograr mantener su independencia, pues una enfermedad controlada mejora la percepción de ausencia de síntomas: 
Yo tomaba muchos remedios para la diabetes, hay una planta que se llama insulina y cubio. Lo que pasa es que las plantas es lo que dejó nuestro señor Jesucristo, Dios es quien nos da la enfermedad y nos da la medicina que uno debe de tomar, entonces a veces uno no cree pero hay que tomarlos para ver que hay mejoría. (P05-44)

\section{Control y atención en salud}

Aquí se refleja la autonomía de los participantes para buscar atención cuando identifican que la requieren; así es como estos hombres logran percibir una alteración en sus cuerpos que no les permite desarrollar actividades de la vida cotidiana de manera que deciden buscar apoyo médico: "Yo acudí al médico y le dije yo siento dolor de cabeza, a veces estoy bien y se me va la onda, y el médico pinchó el dedo y salió el grado de azúcar que tenía, y de ahí para acá me dio metformina y ahorita tomo una pastilla nada más” (P06-06).

Con la adquisición de nuevos conocimientos sobre el cuidado y manejo de la enfermedad, los participantes refieren autorregularse con actividades específicas de prevención para evitar crisis. Sin embargo, con el paso del tiempo y el conocimiento de sus cuerpos logran reconocer signos y síntomas de alerta que los ayuda para la toma de decisiones frente a la asistencia a servicio de urgencias: "Pues en el momento, digamos por ejemplo que se le presenta, como se me presentó esta vez, se me presentó un asunto que fue la hipertensión, pero que sufre de alguna congestión o de alguna cosa, entonces eso sí es que obligatoriamente le toca a uno buscar el médico ¿no?” (P02-33).

\section{Sentido y significado del dolor}

Pocas veces se relaciona la diabetes como una enfermedad que genera dolor específicamente; sin embargo, los participantes dicen que su percepción de sentirse enfermos se relaciona con la sensación de dolor. Para ellos, la presencia de dolor indica la gravedad de los síntomas que expresa la enfermedad incluso si esto no se relaciona directamente con la diabetes:

Uno sabe que la diabetes es una enfermedad que no duele, entonces, no siente uno ese temor, pero por ejemplo es diferente ya sentir uno un dolor y que le van a quitar la pierna a uno, porque ahí sí se siente un poco más de temor, porque se agravó la enfermedad. (P02-09)

Como se ha evidenciado, para ellos la diabetes no representa una enfermedad en sí cuando no hay presencia de dolor; no obstante, ante el dolor, estos hombres intentan soportarlo o manejarlo antes de buscar ayuda: "Ya no es normal, un dolor de tres días ya no es normal, para mí es una alerta de que algo está fallándole a uno y ya, cuando le dura tres días ya luego tiene que normalizarse, si no toca buscar la falla, ahí nos toca irnos para urgencias, antes no" (P03-21).

\section{Sentirse enfermo}

Para que los participantes asociaran la diabetes con la sensación de estar enfermos requirieron observar y percibir las complicaciones físicas derivadas de la DM, por ejemplo, dificultades en la cicatrización e incluso disminución de la agudeza visual. Si estas situaciones no se presentaban, les daba la idea de no estar enfermos:

Yo sentía que me agarró una vaina que no podía estar y me fui al hospital, ese día me hicieron el cateterismo, sentí que el mundo se me iba, siente que no puede respirar casi. Yo sentí, como si se obscureciera la vista, eso es lo que siente uno y claro fui e iniciaron esa vaina, me limpiaron y mire que de acá para acá no he sentido nada. (P04-47)

Por otra parte, estos hombres refieren que la diabetes se relaciona con la falta de energía y con el mismo proceso de envejecimiento; puntualizan que por su conocimiento y vivencia de la enfermedad entienden que su metabolismo es diferente y esto hace que se sientan más cansados. Además, con el paso de los años creen 
que la enfermedad los debilita más. Ellos también describen que sus experiencias previas de hospitalización o entrada a los servicios de urgencias se relacionan con la sensación de estar enfermo:

Tal vez un poco la visión, un poco, pero yo digo que como todo ser humano tiene que irse degenerando en ciertas cosas, la verdad, es una de las cosas que uno no se puede comprar con un muchacho y yo tenga 80 y pico, tiene más energía tiene más habilidad para hacer cualquier cosa, como subir las escaleras a tercer piso o poner un bombillo. (P02-18)

\section{Significado de la diabetes en la vida cotidiana}

Para los hombres, el descubrimiento de la diabetes como enfermedad se asoció a eventos dados por la casualidad, en la que asistieron al servicio de salud por rutina o porque hace tiempo no se examinaban y los resultados de sus estudios médicos detectaron la enfermedad. Para ellos, la diabetes es una enfermedad que significa "crueldad", porque debieron realizar cambios profundos en su vida, sumado a la alteración de su imagen y afectación de su autoestima. Hasta antes de conocer sobre la enfermedad, nunca relacionaron sus síntomas con la DM, sino con otro tipo de enfermedades e, incluso, algunos no se percataron de ellos: "Pero de esta diabetes no sabía nada, la conocí aquí por un examen, yo que me acuerde nunca me hice exámenes, allá en el campo se muere la gente y no saben de qué" (P06-24).

Los participantes minimizan la dimensión de la enfermedad debido a que no perciben síntomas, consideran que la DM puede ser controlada y manejada como cualquier otra enfermedad, sin que esta les genere complicaciones para la vida. En general, su percepción del riesgo de la enfermedad se relaciona con su concepción de que no es una enfermedad infecto-contagiosa y, por ende, ni su salud ni las de los demás está en peligro:

Tantas enfermedades que hay y que ve uno gente que sufre por eso y gente que lo trata de aislar ¿no? Muchas enfermedades contagiosas hacen que lo aíslen en la sociedad... Pero pues lo mío como que no, es como un malecito que me llego ahí, una visita silenciosa que se quedó aquí, y pues que no la he sentido. (P02-73)

La minimización de los síntomas hace que los participantes limiten su concepción de gravedad sobre la enfermedad, lo que implica una menor adherencia al tratamiento, generando fallas en el manejo de la enfermedad, que posteriormente se asocian a un tratamiento según la gravedad:

Porque es que la insulina acaba más a las personas, que a cualquiera que no la consume, ya es una adicción, que tiene que aplicarse morfina en el cáncer es igual a la morfina, que me digan que me van aplicar insulina, y que después se la quitan, después su cuerpo se la va a pedir y exigir, que si le controla, pero su cuerpo se la va a exigir. (P03-43)

En estos casos, el significado que el participante asigna a la diabetes como enfermedad crónica se relaciona con una sensación de devastación y pérdida, además de la aceptación de no haber reconocido y entendido la enfermedad con la importancia que requiere:

Si uno se pone a pensar eso [la diabetes], se acaba uno rápido, si uno piensa en eso todos los días jestá acabado!, toca dejarlo a la voluntad de Dios, cuando Dios lo tenga destinado èya que más? Ya uno vivió su vida bien. Uno también por lo que no se cuidó anteriormente, uno se descompuso completamente. Yo digo que ese mal viene siendo hereditario y además por no cuidarse. (P05-27)

\section{Discusión}

La ausencia o minimización de síntomas característicos de la DM hace que las personas perciban su salud y bienestar como buenos; sin embargo, esto puede llevar a una idea errónea respecto a la importancia que se le da a la diabetes como enfermedad crónica que debe controlarse. Ante esta concepción de salud se hace complejo y difícil el entendimiento de la adherencia al tratamiento y las razones para hacer los cambios en el estilo de 
vida. Frente a estos cambios, no se puede dejar de lado que la familia desempeña un papel fundamental en el control y manejo de la enfermedad.

Un estudio que exploró la experiencia de vivir con DM (23) describió resultados similares a los de esta investigación, en especial lo relacionado con la asimilación y adaptación a la enfermedad; en la que se presentan diferentes etapas donde se puede aprender a vivir con las manifestaciones de la enfermedad, hasta llegar a la adaptación. Se menciona especialmente la familia: vivir con sus miembros tiene efectos positivos en los regímenes de comportamiento, más que en los regímenes de medicación de la diabetes.

Desde otra perspectiva, esta investigación fue particularmente interesante respecto al enfoque de género que muestra, en especial los aspectos relacionados con el autocuidado y los estilos de vida de los participantes. Los resultados evidenciaron características propias de la población masculina adulta mayor, en la que hacer los cambios en la alimentación y la adherencia al tratamiento de manera independiente es complejo, porque representa una modificación de una cotidianidad de muchos años, sumado a la mínima atención que presentan sus síntomas y la espera de complicaciones para tomar la decisión de buscar ayuda.

Estudios similares con perspectiva de género abordaron el tema del autocuidado y los estilos de vida en personas con diabetes mellitus. En el primero de ellos, realizado por Trujillo y Nazar (24), identificó que aun cuando más hombres que mujeres buscaban activamente información sobre diabetes, ese mayor conocimiento de los varones no se tradujo en mejor autocuidado, pues suelen negar la enfermedad y rechazar los ajustes en los patrones de alimentación. Este estudio también evidenció que las mujeres participantes eran casi 10 años más jóvenes que los hombres, lo cual sugiere que ellos se demoran más en buscar atención médica.

En cuanto a los estilos de vida, las investigaciones de Rossaneis et al. (25) y Cruz et al. (26) encontraron de forma análoga que los hombres presentan comportamientos menos saludables, porque tienen significativamente menor control alimentario y no se realizaron los exámenes de laboratorio con la frecuencia recomendada ( $\mathrm{RP}=1,47 / \mathrm{IC} 95 \%=1,22-1,77)$. La evidencia descrita respalda los hallazgos de este estudio, donde los hombres participantes expresaron sus dificultades para comprometerse a largo plazo con los cambios en el estilo de vida, más que con la medicación.

Por otra parte, algunas revisiones sistemáticas e integrativas $(27,28)$ sobre la temática del cuidado de la DM y su relación con el género describieron varios hallazgos sobre la prevalencia, el control y las complicaciones entre hombres y mujeres. Así, uno de los artículos revisados informaba que el $22 \%$ de los varones versus el $9 \%$ de las mujeres desconocían su enfermedad ( $\mathrm{p}<0,001)$, sumando a un incumplimiento terapéutico del $48 \%$ en los hombres y un $28 \%$ en las mujeres. Adicional a esto, también aparecen las creencias que subestiman la diabetes y su impacto, la cual solo la hacen visible ante la presencia de complicaciones, particularidades que se demuestran en las categorías descritas en esta investigación.

Esta situación también se refleja en un estudio cualitativo en el que participaron hombres con diabetes que tuvieron la pérdida de miembros inferiores; ellos expresaron que en el momento del diagnóstico subestimaron la información dada por el profesional de la salud, porque en sus cuerpos no sentían nada que fuera indicativo de estar enfermo, lo cual les daba la sensación, hasta cierto punto, de su inexistencia, lo que los llevó a subvalorar sus consecuencias por no asumir conductas de autocuidado (29).

Entre las limitaciones del estudio está la imposibilidad de generalizar los hallazgos a toda la población colombiana, ya que existen aspectos culturales y étnicos que tienen particularidades no exploradas en este estudio, por lo que se requieren más estudios basados en género para ampliar la comprensión de la percepción ante la enfermedad.

En resumen, se deben reconocer las diferencias de género en cuanto a la concepción y autocuidado de la DM, porque esto permite un abordaje integral y diferenciado de acuerdo con las necesidades de cada población. Asimismo, se deben plantear intervenciones de enfermería que propendan hacia una adherencia al tratamiento por medio de acuerdos conjuntos y de forma participativa sobre las metas posibles de lograr a corto plazo con el autocuidado, en especial en población de hombres adultos mayores. 


\section{Conclusiones}

La percepción de estar enfermo en hombres con DM tipo 2 en este grupo de participantes se caracteriza por categorías como: automanejo y autogestión de la enfermedad, concepción de salud y bienestar, estrategias de afrontamiento y adaptación a la enfermedad, control y atención en salud, sentido y significado del dolor, sentirse enfermo y significado de la diabetes en la vida cotidiana.

Los hombres participantes son adultos mayores que consideran la DM como una enfermedad a la que no temen, pero que les ha cambiado por completo la vida. Así, la ausencia de dolor u otros síntomas hacen que crean que la enfermedad está controlada; sin embargo, saben que deben adherirse a un estilo de vida que los obliga a alimentarse diferente y a tomar medicamentos, sumado a la dificultad para aceptar ayuda sanitaria.

\section{Referencias}

1. American Diabetes Association. Diagnosis and classification of diabetes mellitus. Diabetes Care. 2013; 36(Suppl 1):S67-74.

2. Organización Mundial de la Salud. Las 10 principales causas de defunción [internet]; 2016. [Citado 2018 jul 15]. Disponible en: http://www.who.int/es/news-room/fact-sheets/detail/the-top-10-causes-of-death

3. Rey ML. Epidemiología de la diabetes mellitus: El impacto social de la enfermedad [tesis de doctorado]. Extremadura: Universidad de Extremadura, España; 2015.

4. Mora Morales E. Estado actual de la diabetes mellitus en el mundo. Acta Méd Costarric [internet]. 2014;56(2):44-6. Disponible en: https://www.scielo.sa.cr/scielo.php?script=sci_arttext\&pid=S0001-60022014000200001

5. Barengo NC, Tamayo DC. Reported diabetes mellitus prevalence rates in the Colombia Healthcare System from 2009 to 2012: analysis by regions using data of the official information sources. Int J Endocrinol. 2015;2015:[7 pantallas]. https://doi.org/10,1155/2015/946419

6. Córdoba R, Cabezas C, Camarelles F, Gómez J, Herráez DD, López A, et al. Recomendaciones sobre el estilo de vida. Atención Primaria. 2012;44:16-22.

7. Cheatham CT, Barksdale DJ, Rodgers SG. Barriers to health care and health seeking behaviors faced by black men. J Am Acam Nurse Pract. 2008;20(11):555-62. https:/doi.org/10.1111/j.1745-7599.2008.00359.x

8. Perrasse AV, Abad SB, Faciolince S, Hernández N, Maya C, Parra L, et al. El control de la diabetes mellitus y sus complicaciones en Medellín, Colombia. Rev Panam Salud Pública [internet]. 2006;20(6):393-9. Disponible en: https://scielosp.org/article/rpsp/2006.v20n6/393-402/es/\#ModalArticles

9. González JC, Walker JH, Einarson TR. Cost-of-illness study of type 2 diabetes mellitus in Colombia. Rev Panam Salud Pública [internet]. 2009;26(1):55-63. Disponible en: https://scielosp.org/pdf/rpsp/2009.v26n1/55-63/ en

10. Hawkins J, Watkins DC, Kieffer E, Spencer M, Espitia N, Anderson M. Psychosocial factors that influence health care use and self-management for African American and Latino men with type 2 diabetes: an exploratory study. J Mens Stud. 2015.23(2):161-76.

11. Uresti RM, Caballero-Rico F, Vázquez-Vázquez M, Ramírez JA. La coherencia en el desarrollo humano y la salud integral. CienciaUAT. 2013;8(1):26-32. https://doi.org/10.29059/cienciauat.v8i1.4

12. Moreno GA. La definición de salud de la Organización Mundial de la Salud y la interdisciplinariedad. Sapiens [internet]. 2008;9(1):93-102. Disponible en: https://www.redalyc.org/pdf/410/41011135004.pdf

13. Ahola AJ, Groop PH. Barriers to self-management of diabetes. Diabet Med. 2013;30(4):413-20. https://doi.org $/ 10.1111 /$ dme.12105

14. Sobralske M. Machismo sustains health and illness beliefs of Mexican American men. J Am Acad Nurs Pract. 2006;18(8):348-50. https://doi.org/10.1111/j.1745-7599.2006.00144.x

15. Corbin, J. Introduction and overview: Chronic illness and Nursing. En: Bernstain, R, Corbin, J. Chronic illness: Research and theory for nursing practice. New York: Springer; 2001. p. 1-15. 
16. Graneheim UH, Lundman B. Qualitative content analysis in nursing research: concepts, procedures and measures to achieve trustworthiness. Nurse Educ Today. 2004;24(2):105-12. https://doi.org/10.1016/j.nedt.2003.10.001

17. Cáceres P. Análisis cualitativo de contenido: una alternativa metodológica alcanzable. Psicoperspectivas. Individuo y Sociedad [internet]. 2008;2(1):53-82. Disponible en: https://psicoperspectivas.cl/index.php/psicoperspectiv as/article/view/3

18. Martínez C. El muestreo en investigación cualitativa: principios básicos y algunas controversias. Ciênc Saúde Coletiva. 2012;17(3):613-19. https://dx.doi.org/10,1590/S1413-81232012000300006

19. Strauss A, Corbin J. Bases de la investigación cualitativa: técnicas y procedimientos para desarrollar la teoría fundamentada. Medellín, Antioquia: Editorial Universidad de Antioquia; 2002. p. 219-35.

20. Valles M. Técnicas cualitativas de investigación social. Madrid: Síntesis; 1999.

21. Krippendorff K. Content analysis: an introduction to its methodology. 3rd ed. Newbury Park, CA: Sage; 2013.

22. Lincoln Y, Guba E. Establishing trustworthiness. En: Bryman A, Burgués RG, editores. Qualitative research. London: Sage; 1999. p. 397-444.

23. Cortés Barragán B, Tzontehua I, Ramínez Girón N, García Solano B. Experiencia de vivir con un integrante de la familia con diabetes tipo 2. Rev Iberoam Educ Investig Enferm [internet]. 2016;6(3):28-37. Disponible en: https://www.enfermeria21.com/revistas/aladefe/articulo/211/experiencia-de-vivir-con-un-integrante-de-1 a-familia-con-diabetes-tipo-2/

24. Trujillo L, Nazar A. Autocuidado de diabetes: una mirada con perspectiva de género. Estud Demogr Urbanos. 2011;26(3):639-70. http://dx.doi.org/10.24201/edu.v26i3.1377

25. Rossaneis MA, Haddad MC, Mathias TA, Marcon SS. Differences in foot self-care and lifestyle between men and women with diabetes mellitus. Rev Latino-Am Enfermagem. 2016;24:e2761. http://dx.doi.org/1 $0,1590 / 1518-8345,1203,2761$

26. Cruz-Bello P, Vizcarra-Bordi I, Kaufer-Horwitz M, Benítez-Arciniega A, Misra R, Valdés-Ramos R. Género y autocuidado de la diabetes mellitus tipo 2 en el Estado de México. Pap Pobla [internet]. 2014;20(80):119-44. Disponible en: http://www.scielo.org.mx/scielo.php?script=sci_arttext\&pid=S1405-74252014000200005

27. Sandín M, Espelt A, Escolar-Pujolar A, Arriola L, Larrañaga I. Desigualdades de género y diabetes mellitus tipo 2: la importancia de la diferencia. Av Diabetol. 2011;27(3):78-87. https://doi.org/10,1016/ S1134-3230(11)70013-8

28. Ledón L. Impacto psicosocial de la diabetes mellitus, experiencias, significados y respuestas a la enfermedad. Rev Cubana Endocrinol [internet]. 2012;23(1):76-97. Disponible en: http://scielo.sld.cu/scielo.php?script=sci_ar ttext\&pid=S1561-29532012000100007

29. Ledón L, Chirinos J, Hernández JA, Fabré B, Mendoza M. El precio de la transformación: reflexiones desde la experiencia de personas viviendo con endocrinopatías. Rev Cubana Endocrinol [internet]. 2004;15(3). Disponible en: http://scielo.sld.cu/scielo.php?script=sci_arttext\&pid=S1561-29532004000300004\&lng=es

\section{Notas}

* Artículo original de investigación

Conflictos de interés: los autores declaran no tener conflictos de interés.

\section{Licencia Creative Commons CC BY 4.0}

Cómo citar este artículo: Chaparro-Díaz L, Mendoza-Catalán G, Carreño Moreno S, Gutiérrez-Valverde JM. Cómo perciben los hombres tener diabetes mellitus tipo 2: casos en Bogotá. Investig Enferm Imagen Desarr. 2020;22. https://doi.org/10.11144/Javeriana.ie22.cpht

\section{Financiamiento: Ninguno}

